\title{
Vulneración del derecho a la dignidad humana en los centros de rehabilitación
} en Ecuador

\section{Violation of the right to human dignity in rehabilitation centers in Ecuador}

\author{
Yolanda Guissel Calva-Vega \\ us.yolandacalva@uniandes.edu.ec \\ Universidad Regional Autónoma de los Andes, Santo Domingo \\ Ecuador \\ https://orcid.org/0000-0002-9771-6181 \\ Wilmer Carlos Rodríguez-Arizala \\ ds.Wilmercra63@uniandes.edu.ec \\ Universidad Regional Autónoma de los Andes, Santo Domingo \\ Ecuador \\ https://orcid.org/0000-0003-1300-2526
}

Recepción: 15 de septiembre 2021

Revisado: 25 octubre 2021

Aprobación: 15 de noviembre 2021

Publicación: 01 de diciembre 2021 


\title{
RESUMEN
}

El objetivo general del presente estudio fue analizar jurídicamente la vulneración del derecho a la dignidad humana en los centros de rehabilitación en Ecuador. Desde la perspectiva cuantitativa y apoyado en el método de análisis documental. Por medio de la revisión de textos, libros legales como el Código Orgánico Integral Penal, Constitución del República del Ecuador, tratados internacionales, Ley Penitenciaria, entre varias jurisprudencias, tesis y doctrinas, orientó de manera informativa para conocer del tema y verificar la vulneración de derechos que se están dando en contra de las personas privadas de su libertad en el canto de Esmeraldas. Se aplicó una encuesta para lo cual la población tomada en cuenta fue las 1734 personas con prisión preventiva del cual se extrajo una muestra de 1110 PPL del Centro de Rehabilitación de Varones. Se concluye, que existe un retroceso en cuanto a los derechos de las personas privadas de la libertad.

Descriptores: Preso; derechos humanos; prisión; violación de los derechos humanos; derecho internacional. (Palabras tomadas de Tesauro UNESCO).

\begin{abstract}
The general objective of this study was to legally analyze the violation of the right to human dignity in rehabilitation centers in Ecuador. From the quantitative perspective and supported by the method of documentary analysis. Through the revision of texts, legal books such as the Comprehensive Organic Penal Code, Constitution of the Republic of Ecuador, international treaties, Penitentiary Law, among various jurisprudence, thesis and doctrines, it provided informative guidance to learn about the issue and verify the violation of rights that are being given against people deprived of their liberty in the song of Esmeraldas. A survey was applied for which the population taken into account was the 1734 people with preventive detention from which a sample of 1110 PPL from the Men's Rehabilitation Center was drawn. It is concluded that there is a setback in terms of the rights of persons deprived of liberty.
\end{abstract}

Descriptors: Prisoner; human rights; prison; violation of human rights; international right. (words takenfrom UNESCO Thesaurus). 


\section{INTRODUCCIÓN}

Las cárceles son todas aquellas construcciones ocupadas por personas a quienes se les ha comprobado un delito y representan un peligro para la sociedad, recibiendo uno de los peores castigos que puede tener el hombre que es la privación de la libertad. Según José Zamora el termino cárcel, tiene su origen del griego "Lautumbie, cárceles destinadas a los prisioneros de guerra que eran cuevas ya que procedían de antiguas canteras, las más célebres fueron las de Siracusa" (Zamora, 2015, p. 30). Por los años 640 después de Cristo, en Roma como lo indica el autor "El sistema carcelario se caracterizó por unas condiciones infrahumanas. En las que reinaba la suciedad, las tinieblas y el hacinamiento, de hecho, muchos morían en la prisión" (Zamora, 2015, p. 79). En las cárceles de Roma y Grecia lo que menos existía era la humanidad ya que los prisioneros tenían horribles tratos, no solo la perdida de la libertad, sino además torturas, con amputaciones de sus piernas, lengua, brazos, etc., hasta la perdida de sus propias vidas.

Han trascurridos varios siglos y se han realizados acuerdos a nivel global para combatir la sobrepoblación en las cárceles, e incluso se han redactado convenios internacionales a favor de los derechos de las personas privadas de libertad, sin embargo, los esfuerzos realizados no han sido suficiente para cambiar esta realidad. En este sentido, según un informe para la Comisión Nacional de Derechos Humanos de México indica que: "La mayoría de los países de América Latina, tienen superada su capacidad de alojamiento, registrándose casos de sobrepoblación muy grave, lo que configura una flagrante violación a los Derechos Humanos de las personas privadas de la libertad" (Rodriguez, 2015, p. 16).

En este contexto, la situación del sistema carcelario es alarmante, sin embargo, no existe un consenso claro sobre qué es exactamente y cómo se mide el hacinamiento, porque no existe un conjunto de criterios internacionalmente aceptados, el concepto más usado sobre el hacinamiento es aquel que parte de la capacidad instalada de un establecimiento o sistema y su comparación con el número de personas albergadas. Según las Reglas Mínimas de las Naciones Unidas para el Tratamientos de los Reclusos (Reglas de Nelson Mandela) la regla número 13 establece: "Los locales de 
alojamientos de los reclusos, y especialmente los dormitorios, deberán cumplir todas las normas de higiene, particularmente en lo que respecta a las condiciones climáticas y, en concreto, al volumen de aire, la superficie mínima, la iluminación, la calefacción y la ventilación" (Asamblea General de las Naciones Unidas, 2015, p. 8).

En ese orden de ideas, la Corte Interamericana de Derechos Humanos se ha manifestado de manera directa sobre este problema, que vulnera, doblemente los derechos de las personas privadas de libertad, es así que en la sesión 131 del 13 al 14 de marzo del 2008, en el principio XVII se pronunció sobre el hacinamiento, indicando que:

La ocupación del establecimiento por encima del número de plazas establecido, será prohibida por la ley, cuando de ello, le siga la vulneración de Derechos Humanos, esta deberá ser considerada pena o trato cruel o degradante. La ley deberá establecer los mecanismos para redimir de manera inmediata cualquier situación de alojamiento por encima del número de plazas establecidas. (Corte Interamericana de Derechos Humanos, 2008).

En este sentido uno de los derechos que se vulnera en el hacinamiento es la dignidad Humana, entendida como un derecho inherente a todo ser humano, que lleva en sí y que no se le puede quitar, independientemente de las cosas horribles que se le pueda imputar. (Bieri, 2017, pág. 9) en la misma línea de pensamiento La Convención Americana sobre Derechos Humanos en el Pacto de San José en su artículo 5 párrafo 2, indica que: Toda persona privada de la libertad será tratada humanamente y con el respeto debido a la dignidad inherente al ser humano. (Asamblea General de las Naciones Unidas, 1978, p. 3).

Es así que la dignidad humana, integra todos los derechos humanos, los civiles, los políticos, los económicos, sociales y culturales, y cuando se niega alguno de estos derechos, significa por lo tanto el desconocimiento o la negación de la dignidad humana, este elemento es transversal a todos los derechos; es un atributo de todos los seres humanos sin discriminación.

En este sentido, tenemos que el hacinamiento carcelario es un problema discutido a lo largo de la historia, por ello ha venido evolucionando en base al desarrollo de la sociedad, los hacinamientos vulneran además los derechos fundamentales como el 
derecho a la vida, salud de cada individuo que se encuentra cumpliendo una pena en un reclusorio, en suma, esta crisis penitenciaria trasgrede la dignidad humana y genera violencia, insalubridad, drogadicción extorción y hasta el encuentro con la muerte dentro de estas instituciones.

En esta misma línea de ideas el primer Centro penitenciario en el Ecuador fue el Penal García Moreno construido entre 1869 y 1874, este centro guarda muchas historias que reflejan la falta de organización y atención, celdas de 7.6 metros en las que cupieron de 18 a 20 PPL, otros en vez de curarse de las adicciones ahí dentro se enfermaban más, celdas que desafortunadamente ocupaban asesinos, psicópatas, violadores e inclusive personas inocentes sin distinción de la gravedad, peligrosidad y salud mental que presentaba cada uno de ellos.

En la historia penitenciaria del Ecuador se crearon otras cárceles, como la Penitenciaria modelo del Litoral, La Roca, cárceles de mujeres de Quito y Guayaquil, sin embargo, las políticas de los gobiernos de turno no han podido controlar uno de los problemas más críticos que vulnera el derecho a la dignidad de las personas privadas de la libertad, como lo es el hacinamiento. El espacio disponible ha colapsado en varios centros penitenciarios en el Ecuador y en especial en el Centro de Rehabilitación de varones de la provincia de Esmeraldas, las falencias dentro de este centro afectan el derecho a la vida, a un trato digno y decoroso y a la integridad física y psíquica de las personas privadas de la libertad.

En este contexto, según lo que establece la Constitución de la República del Ecuador (2008):

Artículo 35. Las personas adultas mayores [...] Las personas privadas de la libertad [...] y quienes adolezcan de enfermedades catastróficas o de alta complejidad, recibirán atención prioritaria y especializada en los ámbitos públicos y privados [...] El estado prestará especial protección a las personas en condición de doble vulnerabilidad.

En consecuencia, en el hacinamiento carcelario no se hace una diferencia de grupos de edad, todas las personas van de 18 años hasta adultos mayores a cumplir una pena, los adultos mayores se encuentran en situación de doble vulnerabilidad, porque están privados de libertad y superan los 65 años de edad, por lo tanto, requiere 
atención prioritaria por mandato constitucional. Hay personas en las cárceles que tienen enfermedades catastróficas y que están privadas de la libertad, en estos casos, Hay condición de triple vulnerabilidad, por lo tanto, en los centros de Rehabilitación no se está cumpliendo con lo establecido en el artículo 35 de la CRE antes mencionado. La Constitución de la República del Ecuador (2008) indica:

Artículo. 201 El sistema de rehabilitación social tendrá como finalidad la rehabilitación integral de las personas [...] así mismo como de la protección de las personas privadas de libertad y la garantía de sus derechos[..].

Las condiciones en que se encuentran los PPL contradice lo establecido en este artículo, por tanto, el estado debe velar para que se cumplan los derechos de los internos y no exista aglomeraciones que impidan tener una vida digna de un ser humano, con los estándares de un sistema que no afecte la seguridad y salud física, psicológica.

El crecimiento poblacional carcelario en el Ecuador supera el $40 \%$ desde el 2009 hasta el 2019, triplicándose la población carcelaria, provocando el descontrol de los reos, por la mala distribución de los recursos asignados al sistema penitenciario, vulnerando así los derechos fundamentales de los presos del país; el sistema de las cárceles se genera por tres ejes que son: organización y composición, la gestión administrativa y financiera y la situación de la población recluida, las cuales son manejadas de la peor manera por la crisis institucional. (Nuñez, 2004).

Los principios rectores de la ejecución de las penas y las medidas cautelares personales de las personas privadas de libertad lo refieren el artículo 7 del Código Orgánico Integral Penal (2014), refiriendo:

Que se alojaran en diferentes lugares de privación de libertad o en secciones dentro de dichos establecimientos, de acuerdo a su sexo y orientación sexual, razón de la privación de la libertad, necesidad de protección de la vida e integridad de las personas privadas de libertad 0 necesidades especiales de atención. (Asamblea Nacional, 2014, p. 10). 
Por su parte el Reglamento del Sistema de Rehabilitación Social establece:

Artículo 25. Las personas privadas de libertad la máxima autoridad en coordinación con el equipo técnico y de seguridad penitenciaria del centro, organizará y ubicará a las personas privadas de la libertad [...] según su condición jurídica, apremio personal, prisión preventiva, sentencia condenatoria, sexo, adultos de adultos mayores, según el nivel de seguridad, según la contravención, según el tipo penal, dependiendo el comportamiento violento, lugar para personas de atención prioritaria. (Directorio del Organismo Técnico del Sistema Nacional de Rehabilitación Social, 2020).

De acuerdo a todo el planteamiento formulado el objetivo general del presente estudio es analizar jurídicamente la vvulneración del derecho a la dignidad humana en los centros de rehabilitación en Ecuador.

\section{METODOLOGÍA}

Desde la perspectiva cuantitativa y apoyado en el método de análisis documental. Por medio de la revisión de textos, libros legales como el Código Orgánico Integral Penal, Constitución del República del Ecuador, tratados internacionales, Ley Penitenciaria, entre varias jurisprudencias y doctrinas, orientó de manera informativa para conocer del tema y verificar la vulneración de derechos que se están dando en contra de las personas privadas de su libertad en el canto de Esmeraldas. Se aplicó una encuesta para lo cual la población tomada en cuenta fue las 1734 personas con prisión preventiva del cual se extrajo una muestra de 1110 PPL del Centro de Rehabilitación de Varones del cantón Esmeraldas, mismos que son afectados por el hacinamiento existente en el Centro referido.

\section{RESULTADOS}

Luego de la obtención de los resultados se presenta a continuación la tabla 1 que muestra la cantidad de personas privadas de libertad, así mismo en el cuadro 1 los resultados de la encuesta aplicada. 
Tabla 1.

Estadísticas de personas privadas de la libertad y sentenciados desde el año 2017 al 2021.

\begin{tabular}{|c|c|c|c|c|c|}
\hline AÑO & $\begin{array}{c}\text { Personas } \\
\text { privadas de la } \\
\text { libertad con } \\
\text { prisión } \\
\begin{array}{c}\text { preventiva } \\
\text { prevas }\end{array}\end{array}$ & $\%$ & $\begin{array}{c}\text { Personas privadas de } \\
\text { la libertad con } \\
\text { sentencia } \\
\text { ejecutoriada. }\end{array}$ & $\%$ & $\begin{array}{c}\text { Cantidad de } \\
\text { personas privadas } \\
\text { de la libertad }\end{array}$ \\
\hline $\mathbf{2 0 1 7}$ & 667 & $50 \%$ & 677 & $50 \%$ & 1344 \\
\hline $\mathbf{2 0 1 8}$ & 739 & $55 \%$ & 594 & $45 \%$ & 1333 \\
\hline $\mathbf{2 0 1 9}$ & 821 & $55 \%$ & 659 & $45 \%$ & 1480 \\
\hline $\mathbf{2 0 2 0}$ & 941 & $56 \%$ & 745 & $44 \%$ & 1686 \\
\hline $\mathbf{2 0 2 1}$ & 897 & $52 \%$ & 829 & $48 \%$ & 1734 \\
\hline
\end{tabular}

Fuente: Centro de Rehabilitación de varones de Esmeraldas.

\section{Cuadro 1.}

Resultados de la encuesta.

\begin{tabular}{|c|c|}
\hline Preguntas & Respuesta \\
\hline $\begin{array}{l}\text { 1. ¿Seleccione el rango de edad en el } \\
\text { que se encuentra actualmente }\end{array}$ & $\begin{array}{l}\text { El } 49 \% \text { de los encuestados se encuentra en } \\
\text { un rango de } 18 \text { a } 30 \text { años de edad, un } 25 \% \\
\text { entre los } 31 \text { y } 40 \text { años y otro } 17 \% \text { con una } \\
\text { edad que fluctúa entre los } 45 \text { y } 50 \text { años y en } \\
\text { una cantidad menor con un } 8 \% \text { entre los } 51 \\
\text { y } 60 \text { años. Interpretación: La mayor } \\
\text { población carcelaria del Centro de } \\
\text { Rehabilitación de Esmeraldas está } \\
\text { compuesta por personas jóvenes, en plena } \\
\text { capacidad para producir. }\end{array}$ \\
\hline $\begin{array}{l}\text { 2.¿Del listado a continuación } \\
\text { seleccione, porque delito se encuentra } \\
\text { detenido? }\end{array}$ & $\begin{array}{l}\text { El } 53 \% \text { corresponde a delitos contra el } \\
\text { derecho a la propiedad, es decir extorción, } \\
\text { estafa, abuso de confianza, robo, hurto, } \\
\text { abigeato, receptación, y un } 22 \% \\
\text { corresponde a delitos contra la inviolabilidad } \\
\text { de la vida, es decir asesinato, sicariato, } \\
\text { homicidio, que representan los datos más } \\
\text { relevantes. Interpretación: El mayor }\end{array}$ \\
\hline
\end{tabular}




\begin{tabular}{|c|c|}
\hline & $\begin{array}{l}\text { porcentaje de personas privadas de la } \\
\text { libertad, cumplen condenas por delitos } \\
\text { menores sancionados de } 1 \text { a } 5 \text { años de pena } \\
\text { privativa de libertad, pese a ello no se } \\
\text { beneficiaron de medidas sustitutivas. }\end{array}$ \\
\hline 3. ¿Está con prisión preventiva? & $\begin{array}{l}\text { El } 44 \% \text { de los encuestados, se encuentra } \\
\text { detenido con prisión preventiva, frente a un } \\
66 \% \text { que respondió que no. Interpretación: } \\
\text { El alto porcentaje de detenidos con prisión } \\
\text { preventiva, es un factor causal del } \\
\text { hacinamiento en este Centro. }\end{array}$ \\
\hline 4. ¿Qué tiempo lleva detenido? & $\begin{array}{l}\text { El } 48 \% \text { de los encuestados indica llevar } \\
\text { detenidos más de un año y un } 39 \% \text { menos } \\
\text { de un año, frente a otros datos menores y en } \\
\text { menor proporción un } 11 \% \text { entre } 4 \text { a } 7 \text { años } \\
\text { entre los datos más relevantes. } \\
\text { Interpretación. - La sobrepoblación } \\
\text { carcelaria se incrementa por delitos contra la } \\
\text { propiedad, como robo de un celular, } \\
\text { billeteras, gallinas, delitos menores etc. }\end{array}$ \\
\hline 5. ¿Qué tiempo dura su condena? & $\begin{array}{l}\text { El } 44 \% \text { de los encuestados respondió que } \\
\text { dura entre un rango de } 4 \text { a } 7 \text { años, el } 27 \% \\
\text { de } 1 \text { a } 3 \text { años con relación a otros datos } \\
\text { menores. Interpretación. - Las penas } \\
\text { impuestas a gran parte de los detenidos son } \\
\text { menores a los } 7 \text { años, y en muchos de los } \\
\text { casos es inferior a } 3 \text { años. }\end{array}$ \\
\hline $\begin{array}{l}\text { 6. ¿Cuenta con lo necesario para llevar } \\
\text { una vida adecuada dentro de este } \\
\text { Centro de Rehabilitación? }\end{array}$ & $\begin{array}{l}\text { Frente a esta pregunta el } 94 \% \text { de los } \\
\text { encuestados respondieron que no y otro } 6 \% \\
\text { respondió que si tiene lo necesario para vivir } \\
\text { en el Centro de Rehabilitación. } \\
\text { Interpretación. La situación en que viven } \\
\text { las personas privadas de libertad es } \\
\text { precaria, y existe una insatisfacción total por } \\
\text { las condiciones de vida. }\end{array}$ \\
\hline $\begin{array}{l}\text { 7. Seleccione ¿Qué elemento considera } \\
\text { necesario para mejorar su condición de } \\
\text { vida dentro del Centro de } \\
\text { Rehabilitación? }\end{array}$ & $\begin{array}{l}\text { El } 33 \% \text { considera que deben vivir menos } \\
\text { cantidad de personas en la misma celda, } \\
\text { frente a } 24 \% \text { indica que no tiene camas para } \\
\text { dormir, un } 24 \% \text { que solicita agua potable por } \\
\text { que el recurso es escaso, y un } 8 \% \text { que } \\
\text { solicita baterías sanitarias. Interpretación. - } \\
\text { Existen muchas personas viviendo en una } \\
\text { misma celda, algunas personas privadas de } \\
\text { la libertad duramen en el piso porque no } \\
\text { tiene cama, no tiene agua potable de } \\
\text { manera permanente o regular en muchos de } \\
\text { los casos el agua es abastecida por } \\
\text { tanqueros. }\end{array}$ \\
\hline
\end{tabular}




\begin{tabular}{|c|c|}
\hline $\begin{array}{l}\text { 8. ¿Qué cantidad de personas considera } \\
\text { como máximo que deberían permanecer } \\
\text { cumpliendo su condena en una misma } \\
\text { celda? }\end{array}$ & $\begin{array}{l}\text { El } 54 \% \text { considera que más de cuatro, un } \\
24 \% \text { establece que deben estar tres y el } 21 \% \\
\text { que indica que es para una persona. } \\
\text { Interpretación: - En el Centro de } \\
\text { Rehabilitación de Esmeraldas existen } \\
\text { Celdas que se denominan barracones, estos } \\
\text { alojan está } 15 \text { personas privadas de la } \\
\text { libertad, de ahí la respuesta del } 54 \% \text { de los } \\
\text { entrevistados. }\end{array}$ \\
\hline $\begin{array}{l}\text { 9. ¿conoce Ud. si dentro del centro de } \\
\text { rehabilitación social de Esmeraldas } \\
\text { existen áreas para internos de baja } \\
\text { peligrosidad y otras áreas para internos } \\
\text { de alta peligrosidad? }\end{array}$ & $\begin{array}{l}\text { El } 73 \% \text { de los encuestados respondió que } \\
\text { tiene conocimiento, y el } 23 \% \text { que no tiene } \\
\text { conocimiento. Interpretación: No existen } \\
\text { áreas para reclusos de baja peligrosidad y } \\
\text { otras para reclusos de alta peligrosidad, lo } \\
\text { que deriva en una escuela del delito, ya que } \\
\text { se mezclan, por falta de espacio físico. }\end{array}$ \\
\hline 10. ¿cómo considera su alimentación? & $\begin{array}{l}\text { El } 51 \% \text { considera que es de bajo valor } \\
\text { nutricional, frente a un } 35 \% \text { que considera } \\
\text { que no tiene ningún valor nutricional y otro } \\
13 \% \text { que considera de manera mediana la } \\
\text { nutrición y s un } 2 \% \text { indica que es de alto } \\
\text { valor nutricional la alimentación que reciben } \\
\text { en el Centro de Rehabilitación. } \\
\text { Interpretación. - Esto significa que existe } \\
\text { un descontento total, con el tipo de } \\
\text { alimentación sin ningún valor nutricional lo } \\
\text { que atenta contra los derechos a la salud. }\end{array}$ \\
\hline $\begin{array}{l}\text { 11. ¿Con que frecuencia siente que su } \\
\text { integridad personal corre peligro dentro } \\
\text { de este centro de Rehabilitación? }\end{array}$ & $\begin{array}{l}\text { Un } 49 \% \text { indica que es alta, frente a un } 31 \% \\
\text { que dice ninguna y otro } 15 \% \text { que establece } \\
\text { que es mediana por otro lado existe un } 5 \% \\
\text { que indica que es baja? Interpretación. } \\
\text { Según estos datos más de la mitad de la } \\
\text { población carcelaria siente que su integridad } \\
\text { física corre peligro en este Centro de } \\
\text { Rehabilitación. }\end{array}$ \\
\hline $\begin{array}{l}\text { 12.¿Cuántas veces su integridad } \\
\text { personal ha estado en riesgo dentro del } \\
\text { Centro de Rehabilitación de } \\
\text { Esmeraldas? }\end{array}$ & $\begin{array}{l}\text { El } 59 \% \text { indica que no estado en riesgo su } \\
\text { integridad física, un } 23 \% \text { indica que una vez, } \\
5 \% \text { mencionan que tres veces, otro } 5 \% \\
\text { establece } 2 \text { veces, frente a un } 8 \% \text { que dice } \\
\text { que su integridad a estado en riesgo más de } \\
\text { tres veces. Interpretación. - En el caso del } \\
59 \% \text { es posible que no hayan tenido alguna } \\
\text { agresión directa contra su integridad, lo que } \\
\text { tal vez les ocurrió al } 23 \% \text { quienes indica que } \\
\text { su integridad física estuvo en riesgo. }\end{array}$ \\
\hline
\end{tabular}

Fuente: Los autores. 


\section{DISCUSIÓN}

La Constitución del Ecuador establece que las personas privadas de la libertad pertenecen a un grupo de atención prioritaria, el Código Integral Penal (2014):

Artículo 4. Las personas privadas de libertad ...serán tratadas como con respeto a su dignidad como seres humanos. Se prohíbe el hacinamiento.

Pero contraviniendo lo antes mencionado, el hacinamiento es uno de los problemas más graves que atraviesa el Centro de Rehabilitación de Esmeraldas el mismo que tiene capacidad para 1110 personas privadas de la libertad y en la actualidad tiene 1734 lo cual incumple los tratados internacionales de derechos humanos que protegen a las personas privadas de la libertad, pero lo más grave son las condiciones infrahumanas en que viven estas personas, que una vez que entran en estos Centros de Rehabilitación son olvidados por la sociedad, y son sometidas a vivir en un mundo de miseria y desconsuelo.

\section{CONCLUSIONES}

Las personas privadas de libertad del Centro de Rehabilitación de varones de Esmeraldas cumplen su condena en hacinamiento con lo que se vulnera sus derechos constitucionales y humanos como el derecho a la salud, el derecho a la vida, a la educación y a la dignidad humana. En este sentido, las autoridades de control, como son el Ministerio de Gobierno, el Sistema Nacional de Atención Integral a Personas Adultas Privadas de la Libertad (SNAI) y los directores de los centros de rehabilitación están conscientes del hacinamiento y atribuyen este hecho al uso excesivo de la prisión preventiva, por cuanto más del $53 \%$ de las personas privadas de la libertad están con esta medida cautelar.

Por otro lado, los jueces justifican el uso de la prisión preventiva ya que no cuentan con otros medios alternativos como el grillete electrónico, porque el estado no les provee de estos equipos o por la falta de mantenimiento de los mismos, lo que contribuye para que se produzca el hacinamiento. En este sentido, al mantener el Centro de Rehabilitación de Esmeraldas, con hacinamiento el estado ecuatoriano está 
incumpliendo tanto mandatos constitucionales como tratados internacionales, como son: las reglas de Nelson Mandela y el pacto de San José.

Se evidencia que existe un retroceso en cuanto a los derechos de las personas privadas de la libertad ya que en lo formal tanto la normativa ecuatoriana como los tratados internacionales garantizan los derechos de las personas privadas de libertad lo que no se cumple en lo material. El Centro de Rehabilitación de Esmeraldas en cuanto a infraestructura física está diseñado para albergar 1110 personas privadas de la libertad y en la realidad mantiene a 1726 pudiendo colapsar en cualquier momento la estructura física.

\section{FINANCIAMIENTO}

No monetario.

\section{AGRADECIMIENTO}

A la Universidad Regional Autónoma de los Andes, Santo Domingo, por motivar el desarrollo de la Investigación.

\section{REFERENCIAS CONSULTADAS}

Asamblea General de las Naciones Unidas(2015). Reglas Mínimas de las Naciones Unidas para el Tratamiento de los Reclusos (Reglas Nelson Mandela).[United Nations Standard Minimum Rules for the Treatment of Prisoners (Nelson Mandela Rules)] Recuperado de: https://n9.cl/033cy

Asamblea General de las Naciones Unidas. (1978). Convención Americana Sobre Derechos Humanos (Pacto de San Jose).[American Convention on Human Rights (Pact of San Jose)]. Nueva York: Reg. ONU 27/08/1979 No 17955.Recuperado de: https://n9.cl/pp3d0

Asamblea Nacional de la República del Ecuador. (2014). Código Orgánico Integral Penal [Comprehensive Organic Criminal Code]. Recuperado de https://n9.cl/g6sc

Asamblea Nacional Constituyente de la República del Ecuador, (2008). Constitución de la República del Ecuador. [Constitution of the Republic of Ecuador]. Montecristi. Registro Oficial 449 de 20-oct-2008. Recuperado de https://n9.cl/sia 
Bieri, P. (2017). La Dignidad Humana Una manera de Vivir.[ Human Dignity A Way to Live]. Barcelona: Herder Editorial, S.L., Barcelona.

Corte Interamericana de Derechos Humanos. (4 de marzo de 2008). Principios Y Buenas Practicas sobre la Protección de las Personas Privadas de Libertad en las Américas.[ Principles and Good Practices on the Protection of Persons Deprived of Liberty in the Americas]. Recuperado de: Corte Interamericana de Derechos Humanos: $\underline{\text { https://n9.cl/idnx }}$

Directorio del Organismo Técnico del Sistema Nacional de Rehabilitación Social. (2020). Reglamnto del Sistema de Rehabilitación Social.[ Regulation of the Social Rehabilitation System]. Recuperado de: https://n9.cl/crsnacional-12

Nuñez, J. (2004). La crisis del sistema penintenciario en Ecuador.[ The crisis of the penitentiary system in Ecuador]. Recuperado de: https://n9.cl/21qwg

Rodriguez, M. (2015). Hacinamiento Penitenciario en America Latina:Causas y Estrategías Para su Reducción.[ Prison Overcrowding in Latin America: Causes and Strategy for Its Reduction]. Mexico: ISBN:978-607-729-206-7.

Zamora, L. (2015). La Administración Penitenciaria en el Derecho Romano, Gestión, Tratamiento de los reclusos y mejora de la custodia carcelaria.[ The Penitentiary Administration in Roman Law, Management, Treatment of Prisoners and Improvement of Prison Custody]. Madrid: DYKINSON. 